\title{
Valle Giumentina (Abruzzes). Septième mission : étude des comportements techno-économiques au Pléistocène moyen
}

Élisa Nicoud, Valentina Villa, Juliette Guibert-Cardin et Gourguène Davtian

\section{OpenEdition}

\section{Journals}

Édition électronique

URL : http://journals.openedition.org/cefr/2713

DOI : $10.4000 /$ cefr. 2713

ISSN : 2282-5703

Éditeur

École française de Rome

Référence électronique

Élisa Nicoud, Valentina Villa, Juliette Guibert-Cardin et Gourguène Davtian, « Valle Giumentina (Abruzzes). Septième mission : étude des comportements techno-économiques

au Pléistocène moyen », Chronique des activités archéologiques de l'École française de Rome [En ligne], Italie centrale, mis en ligne le 18 avril 2019, consulté le 09 août 2019. URL : http:// journals.openedition.org/cefr/2713; DOI : 10.4000/cefr.2713

Ce document a été généré automatiquement le 9 août 2019

(c) École française de Rome 


\title{
Valle Giumentina (Abruzzes). Septième mission : étude des comportements techno- économiques au Pléistocène moyen
}

\author{
Élisa Nicoud, Valentina Villa, Juliette Guibert-Cardin et Gourguène \\ Davtian
}

\section{NOTE DE L'AUTEUR}

Les recherches à Valle Giumentina sont financées par l'École française de Rome, le laboratoire CNRS CEPAM UMR 7264, l'université Côte d'Azur, la Fondation Pescarabruzzo, le Parco nazionale della Majella, et bénéficie du soutien logistique de la commune d'Abbateggio, de l'Archéoclub de Pescara, du Museo delle Genti d'Abruzzo et de la commune de Caramanico Terme.

Les chercheurs impliqués dans le projet couvrent de nombreuses disciplines de l'étude du Quaternaire. La fouille est dirigée par E. Nicoud.

Membres de l'équipe de recherche et collaborateurs (les chercheurs marqués d'un astérisque sont intervenus sur le site) : Élisa Nicoud*, Valentina Villa*, Daniele Aureli, Marina Pagli, Giovanni Boschian, Silvano Agostini, Christine Chaussé*, Paul Mazza, Maria Adelaide Rossi, Fabio Fusco, Catherine Kuzucuoglu, Biagio Giaccio, Marion Hernandez, Christelle Lahaye, Norbert Mercier, Cristina Lemorini, Andrea Zupancich, Antonin Tomasso, Nicole Limondin-Lozouet, Emmanuelle Stoetzel, Sébastien Nomade, Alison Pereira*, Jean-Jacques Bahain, Amina Vietti, Eleonora Regattieri, Vincent Robert, Clément Virmoux, Juliette Guibert-Cardin*, Gourgen Davtian* avec la collaboration de Tommaso Colonna et le CGT Group, Andrea D’Andrea, Rosario Valentini, Marianna Fusco, Arnaud Mazuy, Alain Carré*, Sabine Sorin-Mazouni, Cesare Fanelli et Michel Grenet. En 2018, les membres de l'équipe de terrain étaient français et italiens, issus de nombreuses institutions : Alain Carré (CNRS CEPAM), Guido Palmerini (università di 
Chieti-Pescara), Errico Pontis (università degli studi di Napoli L'Orientale), Félicien Capellari (université de Perpignan), Morgane Bardey-Vaillant (Museum national d'histoire naturelle), Julie Perrotte (université de Rennes), Lili Boulet, Steven Sinapayen (université de Nice), Brossard Claire, Élodie Delhommeau (université Paris 1 Panthéon Sorbonne), Juliette Guibert-Cardin (université Côte d'Azur). Nous remercions particulièrement Bona Ham, Maurizio, Joseph, Tonino, Camillo et Laura S.

\section{Objectifs et principaux résultats de la mission de terrain 2018}

1 Valle Giumentina est un gisement des Abruzzes du Paléolithique ancien et moyen en plein air, connu grâce aux travaux approfondis des années 1950 menés par le préhistorien A.M. Radmilli et le géologue J. Demangeot ${ }^{1}$. Sept horizons archéologiques avaient été mis au jour en contexte globalement lacustre, à la faveur d'une ravine profonde qui a incisé les dépôts du Pléistocène jusqu'à $25 \mathrm{~m}$ de profondeur. Les recherches pluridisciplinaires entreprises dans le cadre du programme quinquennal de l'École française de Rome (2012-2016) en collaboration avec la Surintendance des Abruzzes ont permis de préciser la géochronologie du gisement, étape importante et nécessaire pour discourir sereinement des problématiques paléo-historiques, notamment au travers de l'étude des industries lithiques. Nos recherches ont réuni plus de 30 quaternaristes et nécessité en premier lieu la mise au jour d'une coupe stratigraphique (VV1) sur $17 \mathrm{~m}$ de puissance, la réalisation d'un carottage mécanique ayant atteint $45 \mathrm{~m}$ de profondeur (VG1), des profils géophysiques et une fouille planimétrique sur une aire de $50 \mathrm{~m}^{2}$. En 2018, les niveaux archéologiques, ALB-42, LDP-41, LABM-40 et SLM-37 ont déjà fait l'objet de fouille manuelle en extension sur une aire allant de $50 \mathrm{~m}^{2}$ à $35 \mathrm{~m}^{2}$, cette diminution étant induite par la mise en place de paliers de sécurité2.

2 Treize niveaux archéologiques sont désormais attestés le long de la coupe VV1. Ils sont compris dans une séquence stratigraphique puissante de $25 \mathrm{~m}$, ayant enregistré deux cycles glaciaires-interglaciaires, bien décrits par les études sédimentologiques et paléoenvironnementales. Les datations par méthode ${ }^{40} \mathrm{Ar} /{ }^{39} \mathrm{Ar}$ sur dépôts volcaniques lui attribuent un âge allant de 620000 ans à la base à 450000 ans pour le dernier niveau d'occupation, lui-même scellé par des dépôts glaciaires et pédogénétiques plus récents ${ }^{3}$. Ainsi, la poursuite des recherches de terrain à Valle Giumentina dans le cadre du nouveau projet quinquennal 2017-2021 de l'École française peut désormais se concentrer sur les données archéologiques.

3 La mission 2018 (10 juin-12 juillet) nous a réservé des surprises stratigraphiques. L'objectif était la fouille d'un ou deux fins horizons marron compris dans l'unité LAC-34 et apparus lors du nettoyage de la coupe VV1 en 2013 et 2015 (fig. 1). Épais respectivement de 1,5 et $8 \mathrm{~cm}$, ils avaient livré alors plusieurs éclats de façonnage bifacial. Ces horizons se trouvent, au niveau de la coupe, à environ 1,50 m sous le niveau SLM-37 fouillé en 2017 sous une importante épaisseur de sables et de limons calcaires (SFL-35/36). En ouvrant l'aire de fouille vers le nord sur une surface de $25 \mathrm{~m}^{2}$, ces sables intermédiaires s'amincissent fortement, l'horizon marron (LAC-34) se développe jusqu'à $1 \mathrm{~m}$ d'épaisseur. Le site correspond en fait à la rive gauche d'un paléochenal incisant des dépôts lacustres et rempli de sables et de limons. Les niveaux lacustres marron ont donc été testés par deux sondages en tranchées perpendiculaires. Ces tranchées ont finalement atteint le 
niveau archéologique ABF-33 sous-jacent, couvert par les dépôts lacustres de LAC-34 et reposant quant à lui sur une épaisse couche de blocs et de galets (CGB1). Le niveau 33 avait été fouillé lors des travaux d'A.M. Radmilli dans les années 1950 et avait livré 400 pièces lithiques. A.M. Radmilli n'a cependant jamais fait mention d'industries entre les niveaux 34 à 36 . Dans la couche ABF-33, 140 pièces lithiques sont apparues, essentiellement des éclats, faisant état de modalités de débitage par surfaces alternées sans préparation des blocs. Il n'y a pas de restes fauniques. Il reste $20 \mathrm{~m}^{2}$ environ de l'aire de fouille à investir dans cette couche (programme 2019). La présence d'un téphra dans la couche ABF-33 permet de la corréler au SIM 13 b (511 $\pm 3 \mathrm{ka}$, méthode ${ }^{40} \mathrm{Ar} /{ }^{39} \mathrm{Ar}$ ), en accord avec la détérioration climatique observée par l'étude sédimentologique et malacologique.

En 2017, la fouille avait été filmée pour les besoins d'un projet financé par l'université Côte d'Azur IDEX Jedi Académie 5. Cela a donné lieu au film documentaire \#inminimismaxima produit par le CNRS CEPAM et réalisé par Pierre Gaignard et Laura Haby, dont l'avant-première a eu lieu à l'Académie de France à Rome le 20 septembre 2018.

Fig. 1 - Coupe stratigraphique VV1 de Valle Giumentina : a. Localisation générale du site ; b. Prise de vue aérienne en drone et indications de l'emplacement des fenêtres stratigraphiques " VV » le long de la ravine ; c. Coupe VV1 et datations par méthode ${ }^{40} \mathrm{Ar} /{ }^{3} 9 \mathrm{Ar}$ des couches encadrant les niveaux LAC-34 et ABF-33; d. Stratigraphie schématique.

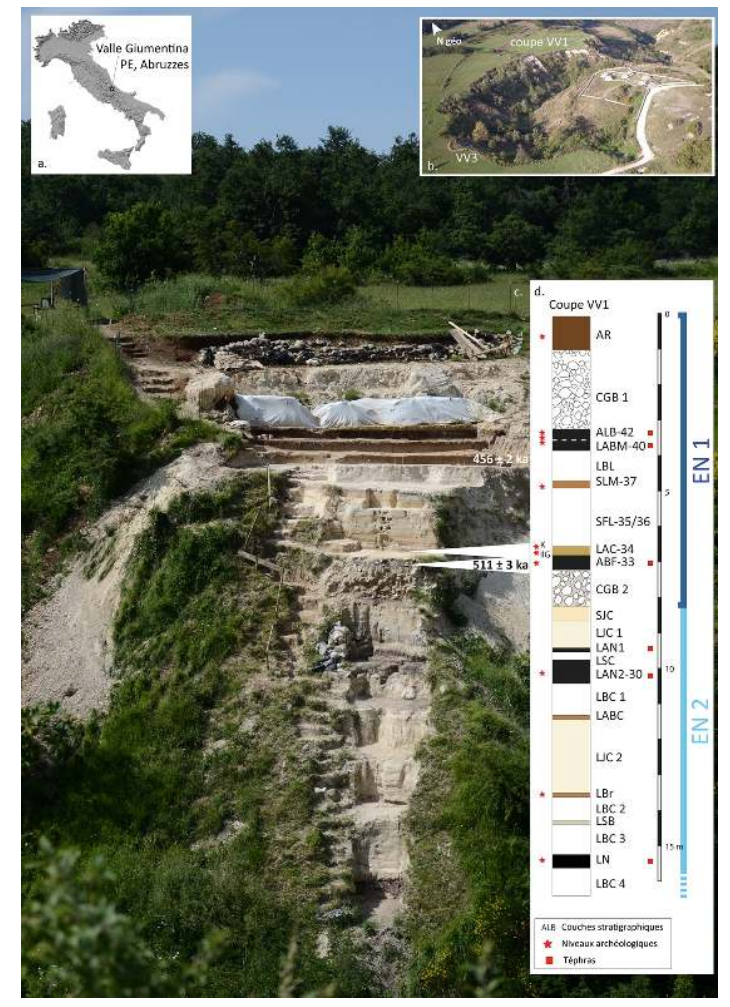

1d. Les flèches blanches indiquent les niveaux ABF-33 et LAC-34 fouillés partiellement en 2018. EN signifie « ensemble».

Cl. EFR/É. Nicoud/P. Gaignard ; c. d'après Pereira 2017, Degeai et al. 2018 ; d. d'après Villa 2017, Villa et al. 2016. 


\section{Contexte stratigraphique et paléoenvironnemental de l'ensemble sédimentaire EN1 et des unités stratigraphiques ABF-33, LAC-34 et SFL-36}

5 La description précise de la séquence stratigraphique est disponible dans différentes publications ${ }^{4}$ et est issue de la thèse de doctorat de Valentina Villa (2017). Les datations par méthodes ${ }^{40} \mathrm{Ar} /{ }^{39} \mathrm{Ar}$ ont été réalisées dans le cadre du doctorat d'A. Pereira (2017) et les études malacologiques par N. Limondin-Lozouet (2017) ${ }^{5}$. L'ensemble EN1 correspond à la dernière phase de sédimentation du bassin de Valle Giumentina (fig. 1-2). Il s'agit d'un ensemble sédimentaire hétérogène, qui comprend des couches carbonatées fluviolacustres, plusieurs paléosols, des dépôts fluvio-glaciaires et des colluvions. Jusqu'à présent, deux retombées volcaniques légèrement remaniées ont été identifiées. La plus ancienne est comprise dans la couche ABF-33 (511 $\pm 3 \mathrm{ka})$, alors que la plus récente se trouve dans les paléosols LABM- 40 et ALB- $42(456 \pm 2$ ka et $456 \pm 5 \mathrm{ka})$.

6 Les données sédimentologiques et micromorphologiques permettent de distinguer trois phases dans l'ensemble EN1. La partie inférieure de l'ensemble (couches CGB2-32 à LAC-34) montre une certaine continuité avec les couches fluvio-lacustres de l'ensemble EN2 sous-jacent. Après un épisode torrentiel correspondant à la couche CGB2-32, une phase de stabilité assez longue est attestée par un paléosol évolué présentant des traits andiques (couche ABF-33, sol sur dépôts volcaniques). Ensuite, la sédimentation fluvio-lacustre reprend de façon temporaire et discontinue avec des dépôts calcaires lités qui encadrent plusieurs horizons bruns faiblement pédogénéisés (couche LAC-34). Dans la partie supérieure de l'ensemble, l'évolution est graduelle. On observe des sédiments limoneux et sableux lités (couches SFL-36 et LBl-38/39) qui, en lame mince, révèlent une double origine. Ces couches sont en effet constituées d'une alternance de dépôts calcaires fluvio-lacustres et éoliens. Ainsi, leur mise en place a été corrélée avec une phase de climat froid et aride. Au sein de cette phase caractérisée par d'intenses apports sédimentaires, la coupe VV1 a enregistré trois phases de stabilité correspondant aux paléosols SLM-37, LABM-40 et ALB-42. La couche CGB1-43 qui suit apparaît comme un diamicton, caractérisé par une matrice calcaire sableuse qui emballe des graviers et des blocs disposés de façon chaotique et très mal triés. Cette couche présente des caractéristiques sédimentologiques similaires à d'autres dépôts morainiques reconnus dans l'Apennin central. Elle pourrait donc signaler un événement froid, en accord avec les dépôts sous-jacents. La succession sédimentaire se termine avec un paléosol polycyclique développé sur des colluvions mises en place en plusieurs temps (couche AR-45/48).

7 Les paléosols qui rythment l'épaisse succession des couches fluvio-lacustres constituant l'ensemble EN1 (couches ABF-33, SLM-37, LABM-40 et ALB-42 dans la coupe VV1; couches ABF1, ABF2 et ALB-LABM dans la carotte VG1) se distinguent par des teneurs plus faibles en $\mathrm{CaCO}_{3}$ que les dépôts détritiques qui les encadrent (0-50\%). Bien que pauvres en matière organique (TOC : 0,2-0,36 \%), les couches ABF-33, LABM-40 et ALB-42 présentent des teintes foncées et une altération marquée (décarbonatation, oxydation et bioturbation). Les processus de pédogenèse ont affecté de façon moins intense la couche SLM, qui apparaît légèrement brunifiée et se distingue des autres par sa richesse en restes de mollusques ${ }^{6}$. Les valeurs de susceptibilité magnétique mesurées le long de 
l'ensemble sédimentaire EN1 sont assez basses, comprises entre 0 et $25\left(10^{-5} \mathrm{SI}\right)$. Leurs variations, assez irrégulières, ne sont pas clairement corrélables aux modifications pédolithologiques. En effet, on pourrait s'attendre à des pics importants de susceptibilité magnétique au niveau des paléosols, d'après les traits d'altération et d'oxydation qui les caractérisent. Or, ces horizons pédologiques montrent la même gamme des valeurs que les couches carbonatées qui les entourent.

Fig. 2 - Synthèse des données géochronologiques, biochronologiques, paléoenvironnementales et paléoclimatiques obtenues à Valle Giumentina.

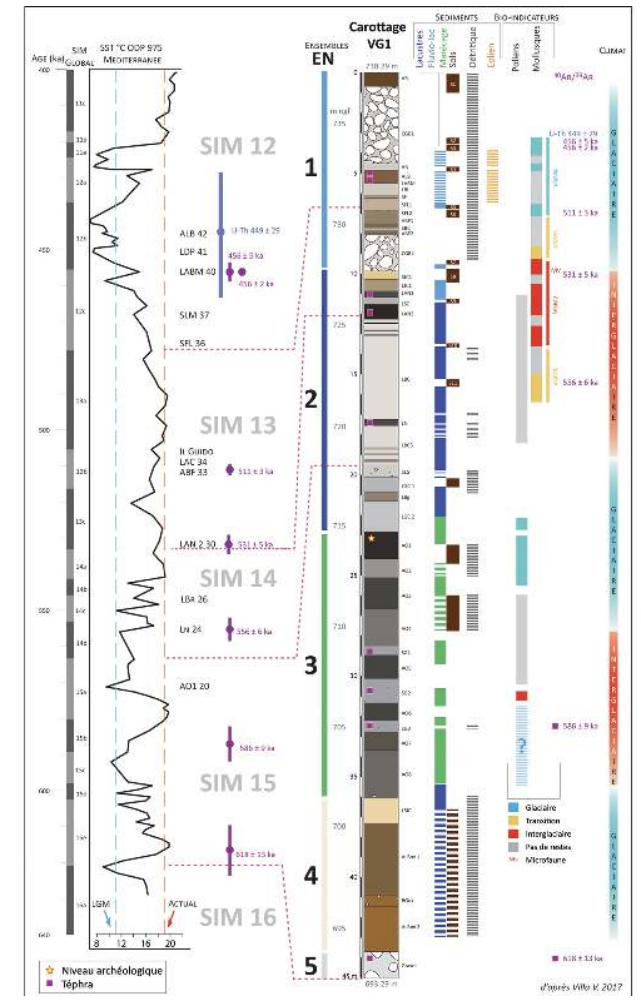

D’après Villa 2017, Degeai et al. 2018 ; courbe isotopique marine dans Girone et al. 2013.

8 Les informations paléoenvironnementales obtenues grâce aux analyses sédimentologiques sont enrichies et complétées par l'étude des malacofaunes conservées à l'intérieur de la séquence sédimentaire de la coupe VV1 (fig. 2). D'après l'étude malacologique l'ensemble EN1 comprend des assemblages assez homogènes, regroupés dans une biozone dite VGM4 (couches ABF-33 à ALB-42). Cette biozone est caractérisée par le renforcement des espèces xérophiles déjà identifiées dans les ensembles sédimentaires sous-jacents. Dans le même temps, des nouvelles espèces calcicoles comme Cernuella cisalpina, Granaria frumentum, Chrondrula tridens et Jaminia malatestae font leur apparition. Ainsi, la biozone VGM4 témoigne de l'établissement progressif d'un milieu très aride, où quelques zones de végétation herbacée xérophile se développent sur des sols calcaires ${ }^{7}$. Les études pluridisciplinaires réalisées sur les dépôts de l'ensemble EN1 concordent sur son attribution à une phase climatique aride et froide, de type glaciaire. De plus, les datations ${ }^{40} \mathrm{Ar} /{ }^{39} \mathrm{Ar}$ réalisée sur les téphras identifiés dans les couches $\mathrm{ABF}$ et ALB contraignent sa chronologie entre environ 510 et $450 \mathrm{ka}$, indiquant donc une corrélation avec la deuxième moitié du MIS 13 et le MIS 12 . 
9 La mission de terrain de cette année a eu pour objet la fouille des niveaux archéologiques ABF-33 et LAC-34, situés à la base de l'ensemble EN1. Les couches sédimentaires qui comprennent les vestiges archéologiques avaient déjà été identifiées lors des premières opérations sur la coupe VV1. La couche ABF-33 apparaissait comme un paléosol brunnoir, très organique, développé sur environ $50 \mathrm{~cm}$ d'épaisseur. La couche LAC-34 se présentait quant à elle comme un ensemble épais de $20 \mathrm{~cm}$ environ, caractérisé par l'alternance de fins lits centimétriques bruns organiques et carbonatés blancs (fig. 3a et c ).

10 Le décapage réalisé pour atteindre la base des dépôts sableux constituant la couche SFL et créer la plateforme de fouille a comporté le recul de la coupe VV1 d'environ 1,5 m vers le nord de référence (nord-ouest géographique). Puis, l'avancement de la fouille archéologique dans les carrés K12 à M12 a mis au jour une nouvelle coupe perpendiculaire à la VV1. L'ensemble de ces opérations a permis une meilleure observation des dépôts et de leur géométrie par rapport aux missions précédentes. En effet, la fouille archéologique se situe à proximité de la marge du bassin de Valle Giumentina, là où les couches qui constituent son remplissage remontent vers les bords et se pincent ${ }^{8}$. Par conséquent, en se déplaçant de l'est vers l'ouest, du bord vers le centre du bassin, les couches sédimentaires se dilatent progressivement et leur lecture est donc plus facile.

11 Les observations les plus intéressantes concernent en particulier la couche LAC, qui montre une épaisseur globale qui varie entre $70 \mathrm{~cm}$ et $1 \mathrm{~m}$. Elle correspond en réalité à un ensemble assez hétérogène, constitué du bas vers le haut des unités suivantes (fig. $\mathbf{3 b}$ ) :

1. Graviers calcaires millimétriques (diamètre max. $1 \mathrm{~cm}$ ) emballés dans une matrice sablo-limoneuse gris clair ;

2. Limons sableux moyens brun clair caractérisés par des nombreuses mouchetures millimétriques noires et quelques poches ( $20 \mathrm{~cm}$ de longueur sur $10 \mathrm{~cm}$ d'épaisseur) de sables fins blancs ;

3. Limons argileux gris et blanc lités ; on distingue deux sous-unités : 3a) limons blanc alternés à des fins lits limono-sableux discontinus et passées sableuses constituées d'un niveau blanc à la base et d'un niveau noir en haut ; $3 b$ ) limons gris clairs à lits discontinus blancs ;

4. Lits sableux millimétriques; de couleur rouille et plus épais à la base dans la partie externe (sud de réf.) de la coupe ; plus fins, discontinus, blancs à la base et noir concrétionnés en haut vers le carré M12 (nord de réf.) ;

5. Limons argileux bruns, litage discontinus plus ou moins foncés ; vers la base lits clairs discontinus ;

6. Limons lités horizontaux ; alternance millimétrique de lits gris et blancs ; traces de bioturbation;

7. Limons argileux bruns massifs, assez hétérogènes ; la moitié inférieure présente une coloration plus foncée par rapport à la moitié supérieure ; on distingue des passées diffuses de sables fins-moyens oranges; limite supérieure correspondante à une surface d'érosion.

8. Lit sableux subhorizontal discontinu de couleur rouille-orange; plusieurs grains minéraux visibles à l'œil nu ; vers la partie méridionale de la coupe, la couche s'épaissit et est constituée de lits sableux millimétriques parallèles, orange et blancs alternés, avec un pendage nord-sud (de réf.) marqué ; observée le long de la coupe I9-I11 la couche apparaît caractérisée par des structures sédimentaires de type rides (fig. 3d). 
9. Limons argileux bruns massifs, assez hétérogènes ; on distingue des lits centimétriques discontinus horizontaux plus clairs et quelques passées sableuses ; limite supérieure érosive.

La succession sédimentaire se poursuit avec les dépôts de la couche SFL, qui est constituée à la base de lits sableux millimétriques de couleur rouille-orange. On observe une alternance entre lits de sables fins et sables moyens concrétionnés, probablement par des carbonates secondaires.

Fig. 3 - Aire de fouille 2018 à Valle Giumentina : a. Vue photogrammétrique de l'aire de fouille 2018. On observe la rive gauche d'un paléochenal ; $b$. Coupe stratigraphique sud-nord (selon le nord de référence) ; $c$. Photographie de la succession des couches ABF-33, LAC-33 et SFL-35 sur la coupe VV1 en 2015 ; d. Vue rapprochée des rides (ripples) ; e. Prélèvements pour l'étude sédimentologique, micromorphologique et chronologique.

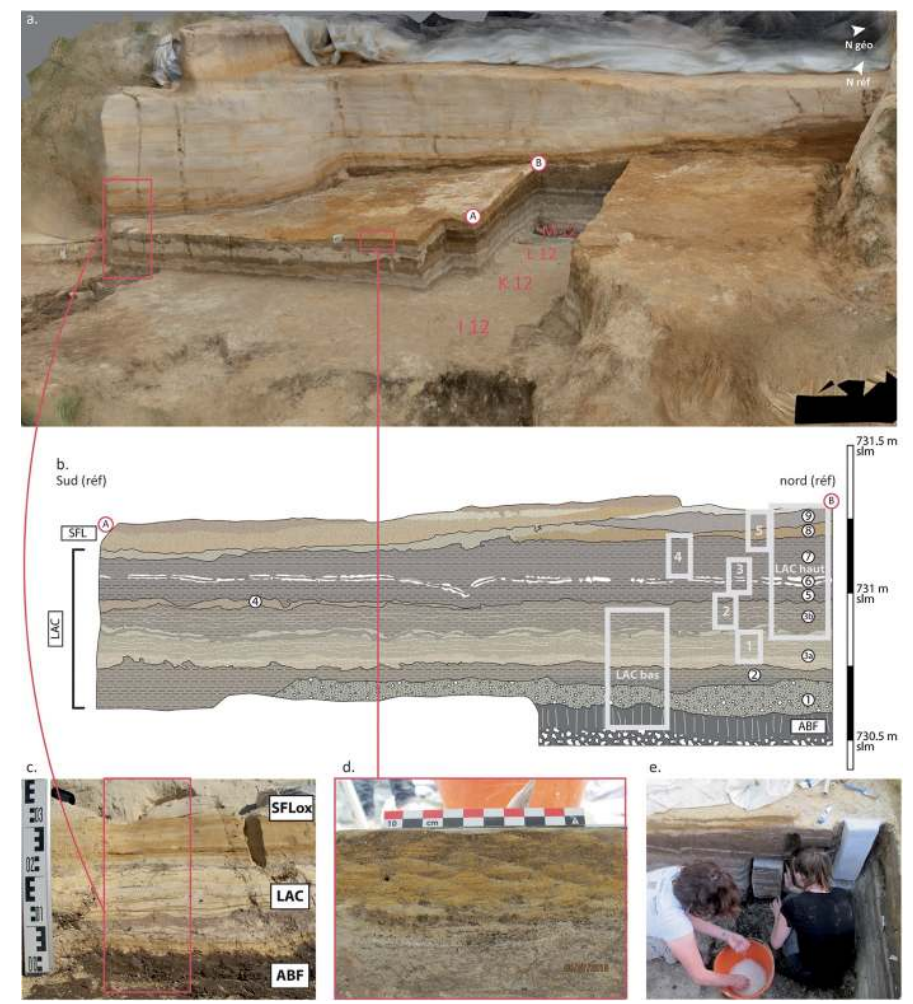

G. Davtian, V. Villa, É. Nicoud.

13 La succession sédimentaire mis au jour au cours de cette campagne a fait l'objet d'échantillonnage pour plusieurs études. Tout d'abord nous avons prélevé des échantillons sédimentologiques pour vérifier l'éventuelle présence de téphras dans certains niveaux sableux des ensembles LAC et SFL qui ont attiré notre attention (texture et minéralogie). Puis nous avons prélevé en deux blocs plâtrés l'intégralité de l'ensemble LAC (fig. 3b et e), de la base de la couche (10) jusqu'au sommet du paléosol ABF. Ces blocs ont permis de réaliser des mesures de susceptibilité magnétique en laboratoire et feront l'objet d'un sous-échantillonnage fin systématique (un échantillon tous les centimètres pour chaque lit) pour analyses sédimentologiques et géochimiques qui seront réalisées au cours de l'année 2019. Les informations issues de ces analyses seront complétées par l'étude micromorphologique des cinq blocs prélevés dans les couches (3a) à (9).

En outre, des prélèvements ont été réalisés dans les sols développés sur téphras encore visibles dans la coupe VV1 (ABF, LAN 2 et LN), le but étant de garder un témoin de ces 
sédiments volcaniques qui pourraient être ultérieurement analysés pour établir des comparaisons et des corrélations stratigraphiques avec d'autres sites et/ou bassins tectoniques d'Italie et d'Europe.

La variété de ces faciès sédimentaires témoigne d'une évolution environnementale complexe, marquée par l'alternance de milieux humides et subaériens. Après la phase de stabilité signalée par le paléosol ABF, la couche de graviers et sables calcaires (1) atteste la reprise d'une sédimentation de type fluviatile produite par des courants assez forts. Dans un deuxième temps, la compétence du courant se réduit et détermine la mise en place d'une couche limono-sableuse (2). Les traits de bioturbation et d'altération qui caractérisent ces dépôts (décarbonatation et oxydation confirmées par les valeurs de susceptibilité magnétique entre 15 et 20, fig. 4) ainsi que leur couleur brune indiquent une nouvelle phase de stabilité et le développement d'une pédogénèse modérée. La couche (2) apparait partiellement érodée dans sa partie sommitale, où elle présente une limite nette et ondulée avec les dépôts sus-jacents.

Fig. 4 - Mesures de susceptibilité magnétique réalisées sur les dépôts de l'ensemble LAC.

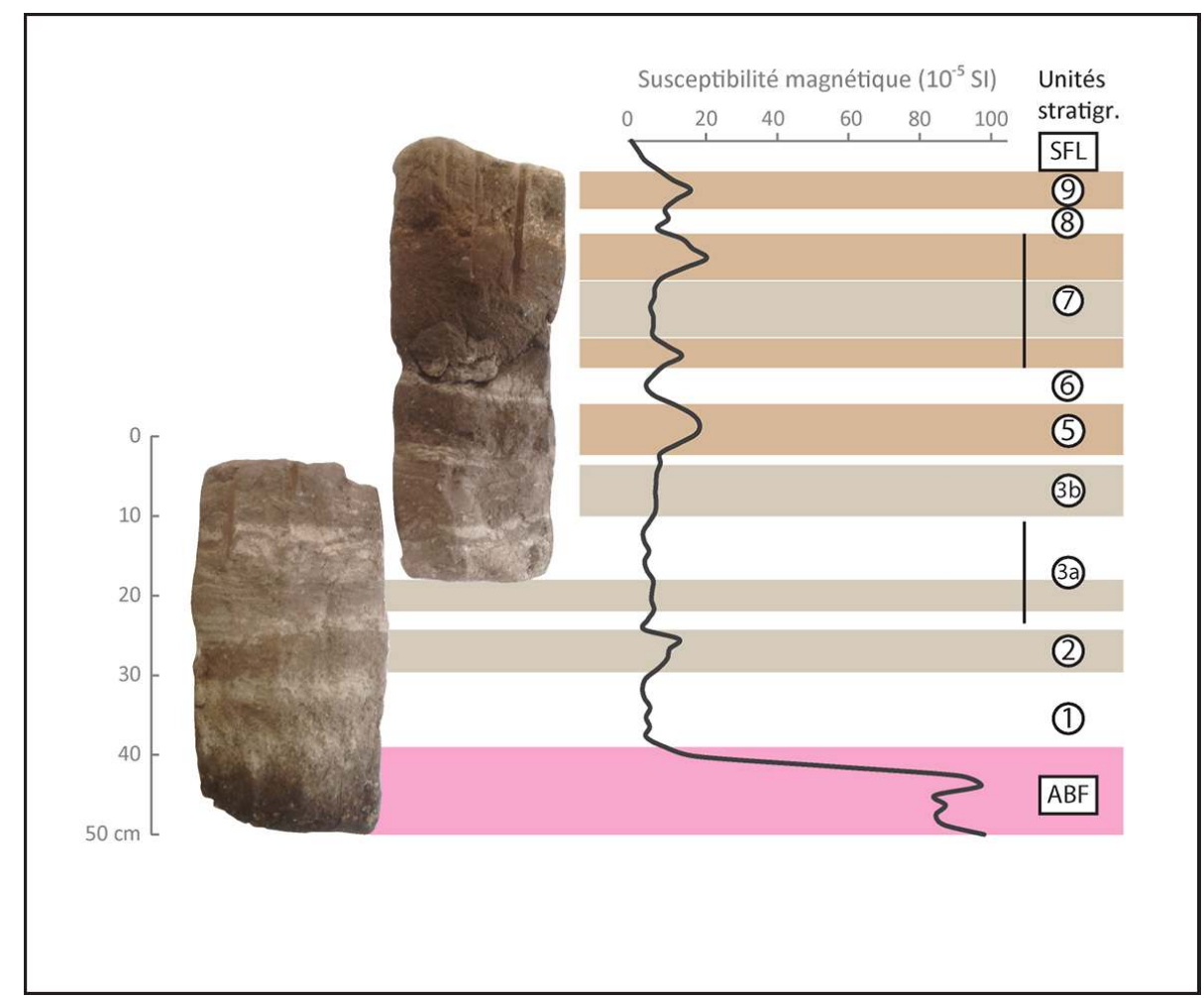

Les bandes beiges indiquent les couches pédogénéisées; la bande rose identifie le sol andique ABF. Cl. V. Villa.

Après cette phase d'érosion, l'ensemble LAC enregistre l'installation d'un environnement de type lacustre qui entraine une sédimentation fine, argilo-limoneuse - couches (3a) et (3 b) - rythmée par l'alternance de fins lits parallèles bruns et blancs. Ces litages horizontaux sont localement très perturbés, affichent des interruptions et des convolutions qui les apparentent à des sismites. Ils étaient sans doute gorgés d'eau quand une perturbation externe, très probablement une forte secousse/séisme s'est vérifiée. Les résultats de susceptibilité magnétique obtenus pour ces niveaux montrent un signal légèrement plus fort des niveaux foncés par rapport aux lits clairs, mais nous ne pouvons 
pas préciser pour l'instant la nature de ces différents dépôts. Les observations micromorphologiques nous apporteront sans doute plus d'éléments.

La couche (4) atteste d'une courte phase d'apports détritiques sableux couverts par une succession de limons argileux bruns finement lités correspondant aux couches (5) et (7). Ces dépôts fins affichent des traits d'altération et de bioturbation modérément développés, semblent avoir subi des processus de pédogenèse et pourraient donc témoigner d'une nouvelle période d'assèchement du bassin de Valle Giumentina. Cette hypothèse doit être vérifiée par l'étude micromorphologique, mais pour l'instant les mesures de susceptibilité magnétique confortent nos observations sur le terrain. Les valeurs sont comprises entre 5 et 21 et permettent d'identifier au moins trois horizons pédologiques distincts (un dans la couche 5 , deux horizons dans la couche 7 ; fig. 4). Les analyses permettront également d'éclaircir l'origine et la composition de la couche limoneuse blanche litée (6) qui sépare les couches (5) et (7). Au vu de son aspect régulier tout le long des coupes stratigraphiques, sur le terrain nous avions proposé l'hypothèse que sa mise en place pouvait être liée à une courte phase de sédimentation lacustre ou fluviatile ou alors au dépôt d'un téphra. En effet, dans cette partie de l'ensemble LAC nous avions observé plusieurs minéraux de couleur noir/vert foncé et transparents visibles à l'œil nu qui nous rappelaient les clinopyroxènes et les sanidines identifiés dans les niveaux téphriques de la séquence sédimentaire de Valle Giumentina. Les mesures de susceptibilité magnétique semblent pour l'instant écarter l'hypothèse qu'il puisse s'agir d'un téphra. Les valeurs sont très basses, proches de 0 , et indiquent plutôt une composition carbonatée de ces dépôts. Toutefois, une étude minéralogique au microscope binoculaire est prévue pour vérifier l'éventuelle présence de verres volcaniques.

Après l'arrêt de la sédimentation qui produit la pédogenèse de la couche (7), une nouvelle phase d'apports détritiques sableux est attestée par la couche (8), puis on observe encore des dépôts limoneux bruns lités et pédogénéisés (9). Ces deux couches sont recoupées par une surface d'érosion très évidente qui présente un pendage ouest-est (de réf.) d'environ $30^{\circ}$. Il s'agit de la limite inférieure d'un chenal qui entaille l'ensemble LAC et que nous avons pu suivre sur plusieurs mètres, sur toute la plateforme créée par la fouille archéologique. Le remplissage de ce canal (10) est constitué de dépôts sableux lités, carbonatés, souvent oxydés, structurant des figures sédimentaires de type rides (fig. 3d), qui permettent d'identifier le sens de l'écoulement vers la ravine actuelle.

Ces dépôts correspondent à la base de la couche SFL précédemment identifiée dans la coupe VV1 et dont la mise en place a été attribuée à un milieu de sédimentation fluviatile puis à des apports éoliens au cours d'une phase de climat froid et aride. La fouille archéologique se trouve donc en rive gauche d'un paléochenal dont l'incision et le remplissage se sont réalisés après $510 \mathrm{ka}$ (chronologie du paléosol $\mathrm{ABF}$ ), entre la fin du MIS 13 et le début du MIS 12.

\section{Fouilles archéologiques et découvertes}

Dans le locus VV3, situé à l'entrée de la ravine et exploité depuis 2015 à la faveur d'un glissement de terrain, il était envisagé de fouiller la couche à limons blancs SFL-36 suite à la découverte en fin de campagne 2017 de quelques industries contenues sous le niveau SLM-37. Malgré l'important effort fourni pour nettoyer les coupes et l'aire de fouille couvertes de colluvions hivernales en VV3, l'extension de la fouille de part et d'autre des 
industries trouvées en 2017 en SFL-36 n'a absolument rien donné. Les bâches de protection et le géotextile qui couvraient ce locus ont ensuite été ôtés : l'érosion annuelle est trop importante et la mise en sécurité n'est plus assurée pour la suite.

Dans le locus principal VV1, une plateforme d'environ $55 \mathrm{~m}^{2}$ se réduisant progressivement pour permettre la mise en place de paliers de sécurité, a été aménagée depuis 2013. Les niveaux archéologiques supérieurs ALB-42, LDP-41 et LABM-40 ont été fouillés ces dernières années et le niveau SLM-37 a fait l'objet d'une fouille fine sur $35 \mathrm{~m}^{2}$ en 2017. L'objectif de la campagne 2018 était la fouille des fins niveaux archéologiques sous-jacents appartenant à l'unité LAC-34 (surnommés " Il Guido/IlG » et "Kordian/K » en l'attente d'une définition plus précise), reconnus lors du nettoyage de la coupe VV1 en 2013, en 2015 et à l'ouverture de la fouille cette année. Les niveaux à fouiller en VV1 n'ont pu être atteints qu'après un décaissement des sables et limons plus ou moins indurés et épais de $60 \mathrm{~cm}$ à $1,50 \mathrm{~m}$, sur une surface de $30 \mathrm{~m}^{2}$ (dont $25 \mathrm{~m}^{2}$ pour l'aire de fouille). Ce travail de terrassement n'a pas pu être réalisé par des moyens mécaniques en raison des intempéries, il a donc été effectué à la main.

La découverte de la portion de rive gauche d'un paléochenal en place des sables observés dans la coupe de référence, nous a amené à revoir nos objectifs. Les sables intermédiaires (SFL-35/36) s'amincissent fortement au nord de l'aire de fouille qui se développe désormais sur $25 \mathrm{~m}^{2}$, l'horizon marron (LAC-34) se développe jusqu'à $1 \mathrm{~m}$ d'épaisseur ( fig. 3). Il fallait donc avant tout bien appréhender ces nouvelles données stratigraphiques et estimer la densité et la concentration des vestiges archéologiques. Il fallait également préparer, dans le respect des règles de sécurité, une aire de fouille assez conséquente pour évaluer le potentiel archéologique des niveaux LAC-34. Or, la rareté des découvertes en LAC-34 et la nécessité d'observer stratigraphiquement le contact inférieur de l'unité LAC-34 nous a engagés à fouiller également quelques mètres carrés du niveau ABF-33 sous-jacent.

Ainsi, seuls 16 éclats sont apparus dans l'unité LAC-34 fouillée essentiellement dans les tranchées $\left(8 \mathrm{~m}^{2}\right)$ et dans sa partie supérieure en M13-14 (fig. 5a). Actuellement l'unité LAC-34 composée de nombreuses couches marron à blanches d'origine lacustre semble compter deux horizons archéologiques, LAC-34-K et LAC-34-IlG (fig. 5b). Le niveau ABF-33 se distingue nettement, à $20 \mathrm{~cm}$ en dessous des dernières pièces de LAC-34. La fouille de la plateforme VV1 sur $8 \mathrm{~m}^{2}$ a livré 114 artefacts lithiques (hors fugaces) dans le niveau ABF-33, essentiellement en silex, quelques-uns en calcaire mais aucun restes osseux ( fig. 5d). Les sédiments résiduels ont fait l'objet de test de tamisage en M12 par quart de $\mathrm{m}^{2}$, non concluant. On note d'ores et déjà une densité assez importante au regard des autres niveaux de Valle Giumentina ainsi qu'un léger pendage vers le nord géographique. Le relevé au GPS différentiel de la zone d'intervention se poursuit, un relevé photogrammétrique a été réalisé en cours de fouille. Par ailleurs l'ensemble des vestiges découverts depuis 2012 fait l'objet d'une base de données exploitable pour un SIG (fig. 5a ). Les vues photogrammétriques sont appréciables étant donné que nous ne disposons pas de recul pour faire des vues d'ensemble de la fouille, en raison du fossé (fig. $\mathbf{5 b}$ ). 
Fig. 5 - Localisation et nature des vestiges découverts à Valle Giumentina (locus VV1) en 2018 : a. Plan des vestiges des niveaux ABF-33 et LAC-34 découverts en 2018 dans le locus VV1 sur une vue orthophotogrammétrique; b. Position altitudinale des vestiges selon les axes du carroyage indiquant un pendage vers le nord géographique ; c. Emprises des différentes fouilles depuis 2012. Le décalage est engendré par les paliers de sécurité ; $d$. Décompte typotechnique des industries.

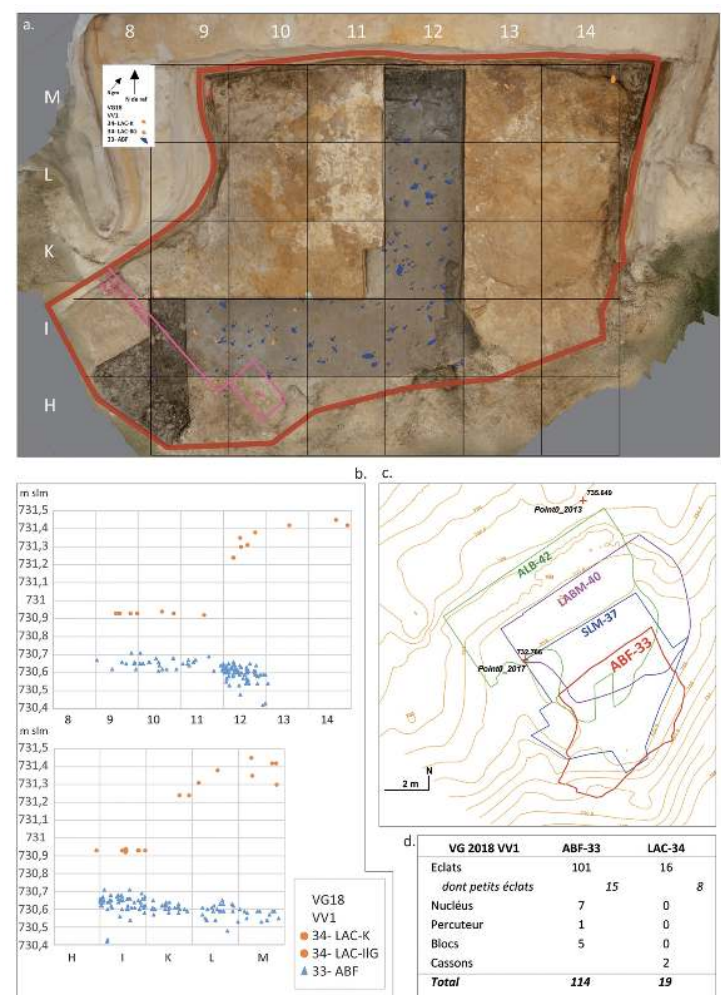

Cl. EFR/É. Nicoud, G. Davtian.

Les études sur l'industrie et la faune se poursuivent en laboratoire, aussi cet exposé est-il extrêmement liminaire. L'industrie du niveau 33 issue des fouilles d'A.M. Radmilli a déjà été observée 9 . Les découvertes de 2018 semblent à première vue s'inscrire dans le même environnement technique (fig. 6). L'intégralité des produits relève du débitage, des éclats épais sont produits, des outils sont présents (racloirs, denticulés). La position du niveau ABF-33 au sommet d'un dépôt à blocs et galets pose la question de l'approvisionnement en matière première. L'étude pétrographique se poursuit. 
Fig. 6 - Exemples d'industries lithiques du niveau ABF-33 lors de leurs découvertes.
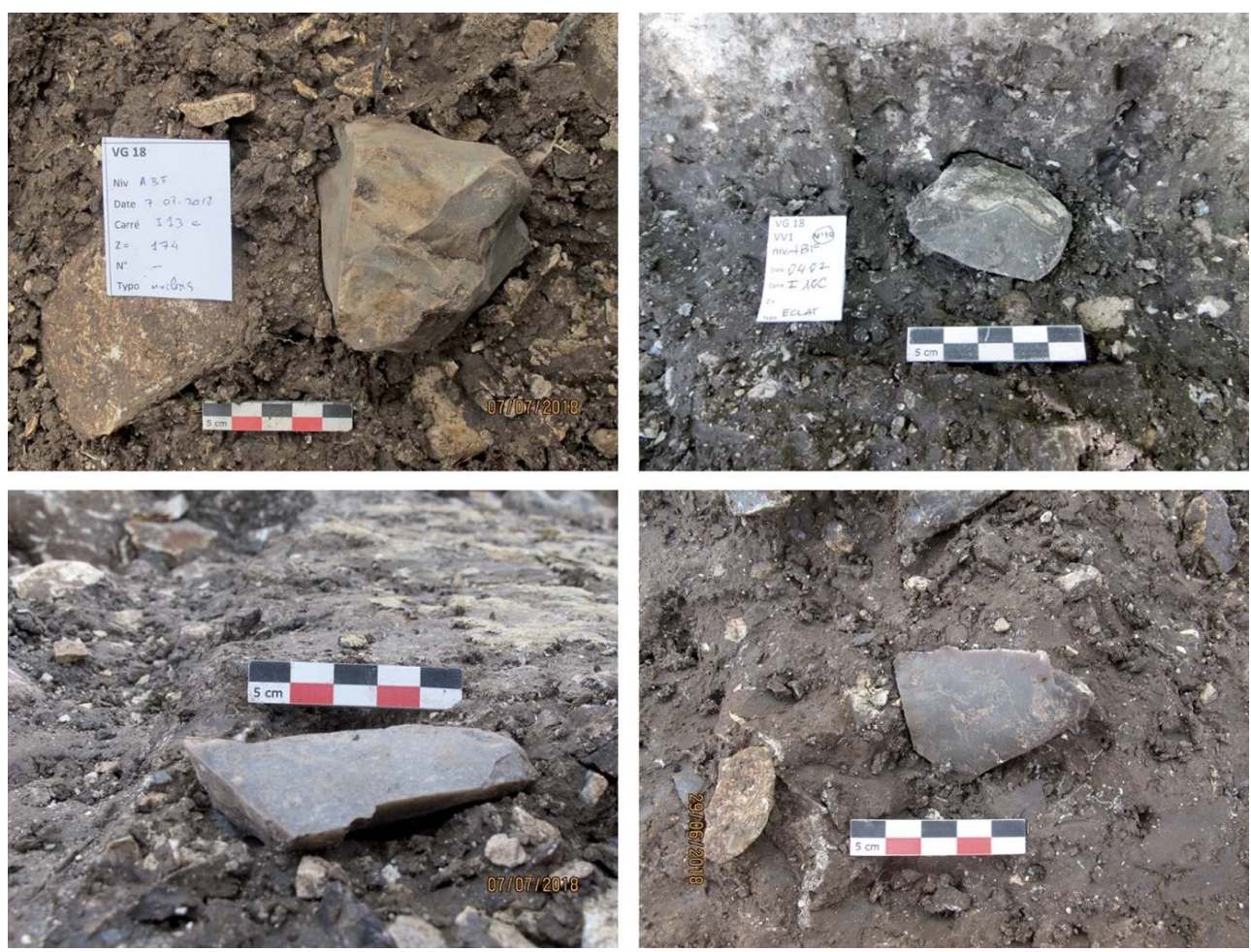

Cl. EFR/É. Nicoud.

Des observations à faible et fort grossissements ont été réalisées afin d'identifier les altérations ou autrement dit les "modifications post-dépositionnelles " affectant les pièces lithiques. Toutes les pièces en silex mises au jour ont été observées. L'état de conservation des artefacts est bon à moyen (fig. 7a-b). Les principales modifications postdépositionnelles sont la patine blanche et le lustré de sol qui affectent la totalité des pièces de l'assemblage. Leur degré de développement est faible à fort. La patine blanche est un voile blanchâtre couvrant une partie ou la totalité de la pièce. Cette altération d'origine chimique témoigne d'un phénomène de dissolution causé par le $\mathrm{pH}$ acide ou basique du sédiment ou par une exposition au solei ${ }^{10}$. Le lustré de sol est une altération rendant la surface du silex brillante. Son origine pourrait être chimique et/ou mécanique. Il pourrait être dû à une dissolution de la silice ou à des micro-mouvements dans le sédiment ${ }^{11}$. Toutes les pièces archéologiques en seraient affectées ${ }^{12}$. Ces deux altérations gênent les observations microscopiques car elles augmentent le réfléchissement de la lumière sur les pièces. Elles peuvent être à l'origine de la disparition des polis d'utilisation. Les pièces qui témoignent de ces altérations ne doivent cependant pas être systématiquement écartées de l'analyse fonctionnelle puisque les macrotraces d'utilisation peuvent toujours être étudiées.

Des micro-enlèvements d'origine taphonomique sont présents sur la moitié des pièces de l'assemblage (fig. 7c). Ils sont parfois accompagnés d'écrasements ou d'émoussés des tranchants et des arêtes. Ces altérations sont d'origine mécanique et peuvent être dues à des mouvements dans le sol, à du piétinement, à de la friction entre deux pièces lithiques, etc. ${ }^{13}$ Les encroûtements sont rares, peu développés et souvent dissociés des tranchants. Ils ne gênent donc que rarement et localement l'observation des tranchants, et n'empêchent pas l'analyse fonctionnelle. Enfin, des bright spots apparaissent lors de 
l'observation au microscope (fig. 7d-e). Ce sont des tâches de poli très réfléchissant. Leur origine est mal connue. Ils pourraient être d'origine mécanique dans le cas d'une friction entre deux silex en présence d'eau ou bien d'origine chimique dans le cas d'un dépôt de silice issue du sédiment ${ }^{14}$. À la surface des pièces du niveau ABF-33, ces bright spots sont peu nombreux et souvent dissociés des tranchants. Ils ne constituent donc pas un problème majeur pour l'analyse fonctionnelle.

Les modifications post-dépositionnelles des pièces lithiques du niveau ABF-33 sont donc d'origine chimique et mécanique. Elles sont en majorité faiblement ou moyennement développées. Les polis d'utilisation sont généralement mal conservés sur des artefacts aussi anciens et les analyses fonctionnelles se basent principalement sur les macrotraces. Les micro-enlèvements d'origine taphonomique constituent donc le principal problème pour l'analyse fonctionnelle de ce niveau car ils sont récurrents. Ils emportent une partie $\mathrm{du}$ tranchant et doivent être distingués des micro-enlèvements fonctionnels ou de la retouche. Les expérimentations sur les micro-enlèvements taphonomiques sont nombreuses et ont prouvé qu'il est possible de les différencier des micro-enlèvements fonctionnels ou de la retouche ${ }^{15}$. Ces micro-fractures n'empêchent donc pas une analyse fonctionnelle et le reste des tranchants est plutôt bien conservé. Cette première expertise taphonomique devra être poursuivie lorsque l'ensemble du niveau aura été fouillé et que toutes les pièces auront été mises au jour. L'analyse fonctionnelle suivra également dans le cadre du doctorat de Juliette Guibert-Cardin (université Côte d'Azur-CEPAM). 
Fig 7 - État de conservation de l'industrie lithique du niveau ABF-33 de Valle Giumentina découverte en 2018 : a. Graphique représentant le degré d'altération des pièces lithiques du niveau ABF-33 (en nombre de pièces). Il est obtenu en cumulant l'ensemble des modifications post-dépositionnelles observées sur les pièces et leur degré de développement ; $b$. Graphique représentant les différentes modifications post-dépositionnelles et la quantité de pièces lithiques du niveau ABF affectées par celles-ci ; c-d-e. Exemples de modifications post-dépositionnelles observées et décrites dans le niveau ABF. $c$. Micro-enlèvement d'origine naturelle avec un écrasement au niveau de l'initiation ce qui n'est jamais observé dans le cas de micro-enlèvements fonctionnels (×10); d. Bright spot (×100); e. Bright spot et lustré de sol $(\times 200)$.
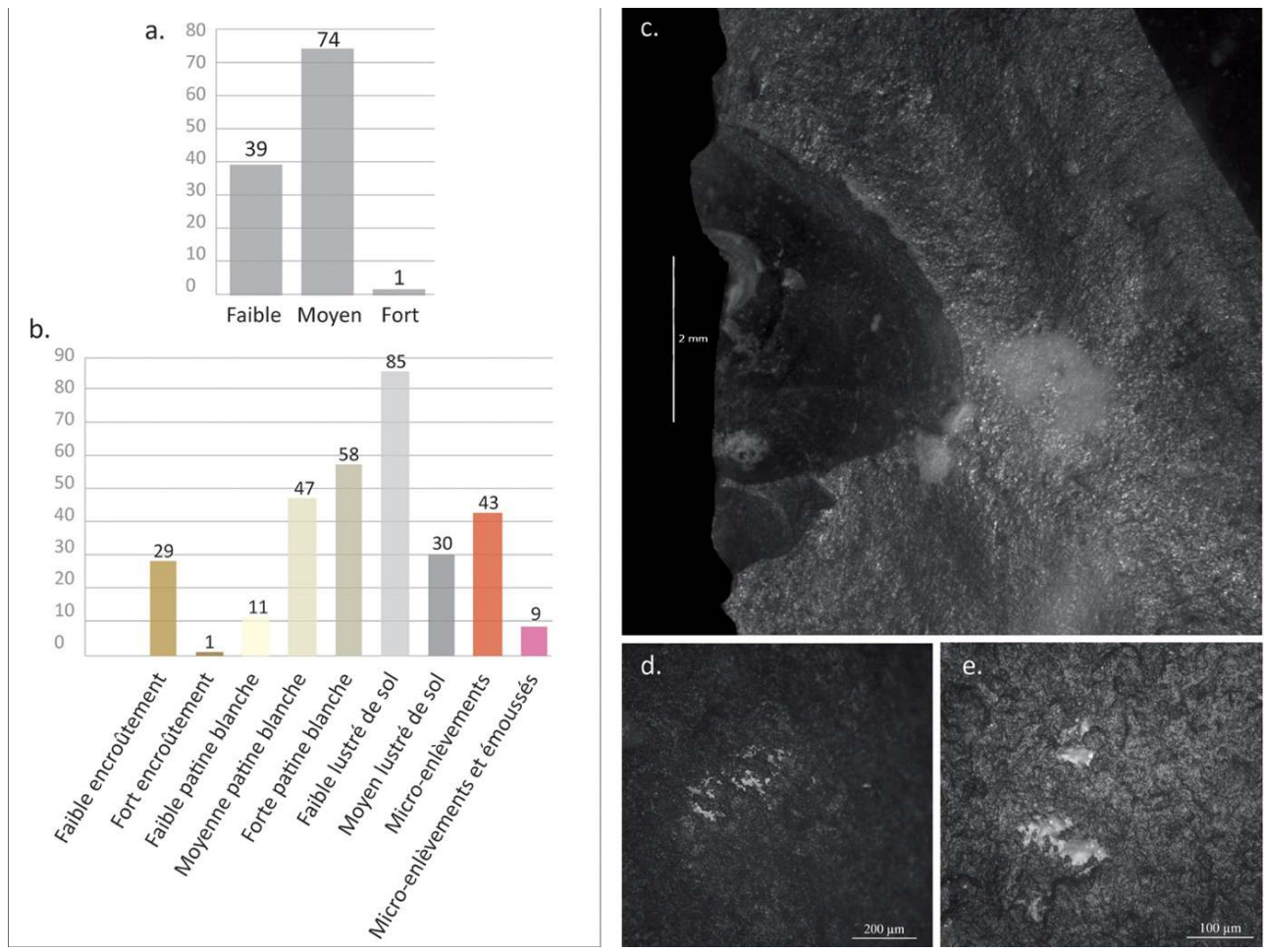

Cl. J. Guibert-Cardin

\section{Perspectives}

L'an prochain sur le terrain, nous envisageons de fouiller le niveau ABF-33 sur toute la plateforme $\left(25 \mathrm{~m}^{2}\right)$. Cela permettra d'obtenir des données conséquentes sur ce cinquième niveau archéologique de Valle Giumentina. Il conviendra en parallèle de poursuivre l'étude sur le mobilier archéologique, en particulier sur l'industrie lithique. Les études technologiques, tracéologiques (thèse de J.Guibert-Cardin), pétrographiques et les dessins ainsi que les analyses spatiales se poursuivent. L'objectif est la rédaction rapide d'un article détaillé sur le niveau supérieur (ALB-42), d'un article sur la micromorphologie des sols en 2019 et d'une monographie sur l'ensemble des travaux dès que possible. Cet important travail de synthèse permettra de mettre en relation les nombreuses données déjà obtenues sur le paléoclimat, le paléoenvironnement et les occupations humaines dans une perspective multiscalaire. Valle Giumentina est une fenêtre rare sur le Paléolithique ancien qui préserve encore sous l'aire de fouille actuelle une séquence archéologique et stratigraphique unique par sa longueur et son âge ancien. Il nous paraît cependant opportun de faire le point sur les nombreuses découvertes de ces dernières années avant de sonder plus profondément ces sédiments. 


\section{BIBLIOGRAPHIE}

Claud $2008=\mathrm{E}$. Claud, Le statut fonctionnel des bifaces au Paléolithique moyen récent dans la Sud-Ouest de la France. Étude tracéologique intégrée des outillages des sites de La Graulet, La Conne de Bergerac, Combe de Brune 2, Fonseigner et Chez-Pinaud/Jonzac, thèse de doctorat, 2008, université Bordeaux I.

Degeai et al. 2018 = J.-Ph. Degeai, V. Villa, C. Chaussé, A. Pereira, S. Nomade, D. Aureli, M. Pagli, É. Nicoud, Chemical weathering of palaeosols from the Lower Palaeolithic site of Valle Giumentina, central Italy, dans Quaternary Science Reviews, 183, 2018, p. 88-109.

Demangeot - Radmilli 1966 = J. Demangeot, A.M. Radmilli, Le gisement paléolithique de Valle Giumentina (Apennin central) et ses problèmes, dans Eiszeitalter und Gegenwart, 17, 1966, p. 159-299.

Girone et al. 2013 = A. Girone, P. Maiorano, M. Marino, M. Kucera, Calcareous plankton response to orbital and millennial-scale climate changes across the Middle Pleistocene in the western Mediterranean, dans Palaeogeography, Palaeoclimatology, Palaeoecology, 392, 2013, p. 105-116.

Lemorini 2000 = C. Lemorini, Reconnaître des tactiques d'exploitation du milieu au Paléolithique moyen : la contribution de l'analyse fonctionnelle. Étude fonctionnelle des industries lithiques de Grotta Breuil (Latium, Italie) et de La Combette (Bonnieux, Vaucluse, France), Oxford, 2000 (BAR International Series, 858).

Levi Sala 1986 = I. Levi Sala, Use-wear and post-depositional surface modification: a word of caution, dans Journal of Archaeological Science, 13, 1986, p. 229-244.

Levi Sala 1996 = I. Levi Sala, A study of microscopic polish on flint implements, Oxford, 1996 (BAR International Series, 629).

Limondin-Lozouet et al. 2017 = N. Limondin-Lozouet, V. Villa, A. Pereira, S. Nomade, J.-J. Bahain, E. Stoetzel, D. Aureli, M. Pagli, É. Nicoud, Middle Pleistocene Molluscan Fauna from Central Italy at Valle Giumentina (Abruzzo): palaeoenvironmental, biostratigraphical and biogeographical implications, dans Quaternary Science Reviews, 156, 2017, p. 135-149.

Mansur-Franchomme 1986 = M.E. Mansur-Franchomme, Microscopie du matériel lithique préhistorique : traces d'utilisation, altérations naturelles, accidentelles et technologiques, Paris, 1986.

Nicoud et al. 2015 = É. Nicoud, D. Aureli, M. Pagli, Comportements techniques au Pléistocène moyen en Italie : nouvelles recherches sur l'industrie lithique et le site de Valle Giumentina (Abruzzes), dans MEFRA, 127-1, 2015. http://journals.openedition.org/cefr/2659

Nicoud et al. 2016 = É. Nicoud, D. Aureli, M. Pagli, V. Villa, C. Chaussé, S. Agostini, J.-J. Bahain, G. Boschian, J.-P. Degeai, F. Fusco, B. Giaccio, M. Hernandez, C. Kuzucuoglu, C. Lahaye, C. Lemorini, N. Limondin-Lozouet, P. Mazza, N. Mercier, S. Nomade, A. Pereira, V. Robert, M.A. Rossi, C. Virmoux, A. Zupancich, Preliminary data from Valle Giumentina Pleistocene site (Abruzzo, Central Italy): a new approach to a Clactonian and Acheulian sequence, dans Quaternary International, 409B, 2016, p. 182-194.

Nicoud et al. 2018 = É. Nicoud, D. Aureli, M. Pagli, Valle Giumentina (Abruzzes, Italie), Sixième mission : étude des comportements techno-économiques au Pléistocène moyen, dans Chronique des activités archéologiques de l'École française de Rome, 2018. http://journals.openedition.org/cefr/1891 
Pereira 2017 = A. Pereira, Apport de la datation ${ }^{40} \mathrm{Ar} /{ }^{39} \mathrm{Ar}$ à la compréhension de l'évolution culturelle des pré-néanderthaliens en Italie centrale et méridionale entre 750 et $250 \mathrm{ka}$, thèse de doctorat, Muséum national d'histoire naturelle, università di Ferrara, École française de Rome, 2017.

Pryor 1988 = J. Pryor, The effects of human trample damage on lithics: a consideration of crucial variables , dans Lithic Technology, 17-1, 1988, p. 45-50.

Van Gijn 1989 = A. Van Gijn, Post-depositional surface modifications, dans Analecta Praehistorica Leidensia, 22, 1989, p. 51-58.

Villa $2017=$ V. Villa, Environnements et occupations paléolithiques d'Italie centrale : la longue séquence pléistocène moyen de Valle Giumentina, thèse de doctorat, université Paris 1 Panthéon-Sorbonne, università di Pisa, École française de Rome, 2017.

Villa et al. 2015 = V. Villa, C. Chaussé, É. Nicoud, D. Aureli, M. Pagli, Valle Giumentina (Abruzzes, Italie) ; nouvelles observations de la stratigraphie, dans MEFRA, 127-1, 2015. https:// journals.openedition.org/mefra/2658

Villa et al. 2016a = V. Villa, A. Pereira, C. Chaussé, S. Nomade, F. Fusco, N. Limondin-Lozouet, J.P. Degeai, V. Robert, C. Kuzucuoglu, G. Boschian, D. Aureli, M. Pagli, É. Nicoud, A MIS 15-MIS 12 record of environmental changes and Lower Palaeolithic occupation from Valle Giumentina, central Italy, dans Quaternary Science Reviews, 151, 2016, p. 160-184.

Villa et al. 2016b = V. Villa, C. Virmoux, C. Chaussé, J.-P. Degeai, V. Robert, C. Kuzucuoglu, G. Boschian, S. Agostini, D. Aureli, M. Pagli, É. Nicoud, The long sedimentary succession of the Valle Giumentina basin (Abruzzo, central Italy): new evidence from stratigraphic studies and electrical resistivity tomography (ERT), dans Alpine and Mediterranean Quaternary, 29-2, 2016, p. 193-199.

\section{NOTES}

1. Demangeot - Radmilli 1966 ; Nicoud et al. 2015.

2. Nicoud et al. 2016 et Nicoud et al. 2018.

3. Villa et al. 2016a ; Villa 2017 ; Pereira 2017 ; Degeai et al. 2018.

4. En particulier Villa et al. 2015 ; Villa 2016a ; Nicoud et al. 2016 ; Degeai et al. 2018.

5. Villa 2017 ; Pereira 2017 ; Limondin-Lozouet et al. 2017.

6. Limondin-Lozouet et al. 2017 ; Nicoud et al. 2018.

7. Limondin-Lozouet et al. 2017.

8. Villa et al. 2016b.

9. Nicoud et al. 2015.

10. Van Gijn 1989.

11. Mansur-Franchomme 1986 ; Levi Sala 1986.

12. Mansur-Franchomme 1986.

13. Lemorini 2000.

14. Levi Sala 1996.

15. Pryor 1988 ; Lemorini 2000 ; Claud 2008. 


\section{INDEX}

Index géographique : Valle Giumentina

institutions Institutions : École française de Rome, Soprintendenza per i beni archeologici dell'Abruzzo, CNRS (CEPAM UMR 7264, ArScAn-AnTeT UMR 7041, LGP UMR 8591, ASM UMR 5140, IRAMAT UMR 5060, LSCE UMR 8212 et HNHP UMR 7194), INRAP, université Bordeaux Montaigne, université Paris 1 Panthéon-Sorbonne (ED de géographie de Paris), Commissariat à l'énergie atomique, Università degli studi di Siena (Preistoria e Antropologia), Università degli studi di Pisa (Biologia), Università degli studi di Firenze (Scienze della Terra), di Roma La Sapienza, CNR, CGT Group Siena, Parco nazionale della Majella, Fondation Pescarabruzzo, Comune di Abbateggio, Comune di Caramanico Terme, Museo delle Genti d'Abruzzo, Archeoclub di Pescara.

Keywords : Abruzzo, Middle Pleistocene, Valle Giumentina, lithic Technology

Mots-clés : Paléolithique, Pléistocène moyen, Valle Giumentina, technologie lithique

\section{AUTEURS}

\section{ÉLISA NICOUD}

CNRS, UMR 7264 CEPAM, université Côte d'Azur - elisa.nicoud[at]cepam.cnrs.fr

\section{VALENTINA VILLA}

Post-doctorante, Laboratoire UMR 8591 LGP Meudon

\section{JULIETTE GUIBERT-CARDIN}

Doctorante, université Côte d'Azur, UMR 7264 CEPAM

\section{GOURGUÈNE DAVTIAN}

CNRS, UMR 7264 CEPAM, université Côte d'Azur 